DOI: 10.1136/annrheumdis-2017-eular.3718

\section{AB0628 MACROVASCULAR DYSFUNCTION OF UPPER AND LOWER LIMBS CORRELATES WITH DIGITAL ULCERATIONS IN PATIENTS WITH SYSTEMIC SCLEROSIS}

E. Moradkhan ${ }^{1}$, S. AleSaeidi ${ }^{2}$, F. Gharibdoost ${ }^{3} .{ }^{1}$ Shahid Beheshti University of Medical Siences; ${ }^{2}$ Rheumatology, Internal medicine, Tehran university of medical sciences, Medical Faculty; ${ }^{3}$ Tehran university of medical siences, Tehran, Iran, Islamic Republic Of

Background: Systemic sclerosis (SSc) is a chronic connective tissue disorder of unknown etiology characterized by tissue fibrosis and vascular damage [1]. Digital ulcerations (DUs) are common manifestations of vascular involvement [2]. Althoughthe vascular dysfunction of SSc has been considered mainly to affect microvasculature [3], there is recent evidence showing that SSc is associated with the prevalence of large vessel disease [4]. However, only a few studies investigated the relationship between macrovascular disease and its role in the clinical manifestations of SSc such as ulcers.

Objectives: To assess the relationship between the Macrovascular dysfunction of upper and lower limbs with digital ulcerations in patients with systemic sclerosis. Methods: Ninety patients with SSc (45 cases with DUs and 45 cases without DUs) enrolled in this study. Patients with other rheumatologic diseases and diabetics patients were excluded from the study. Data which were collected from the patients, included, age, past medical history of cardiovascular disease and dyslipidemia, SSc disease duration, type of SSc (IcSSc or dcSSc), Raynaud's phenomenon (RP), RP duration and digital ulcerations (DUs), Body weight (BW), Height, Waist circumference (WC), Body mass index (BMI), Blood pressure (BP), serum levels of SCL-70 antibody and Anti $\beta 2$ microglobulin antibody. Then Doppler sonographies were performed. The outcome variables were the peak systolic velocity (PSV) and resistance index (RI) of ulnar, radial, popliteal, dorsalis pedis and tibia artery.

Results: The SSc patients with DUs have significantly lower PSV and higher RI in the ulnar (PSV:52. $1 \pm 7.9$ vs $55.7 \pm 4.1, p=0.006$ and $\mathrm{Rl}: 0.58 \pm 0.15$ vs $0.52 \pm 0.06$, $\mathrm{p}=0.003$ ), dorsalis pedis (PSV: $33.9 \pm 1.9$ vs $34.5 \pm 0.4, \mathrm{p}=0.027$ and $\mathrm{RI}: 0.54 \pm 0.11$ vs $0.50 \pm 0.04, p=0.045$ ) and tibial artery (PSV: $32.6 \pm 3.1$ vs $34.4 \pm 0.9, p<0.001$ and RI: $0.62 \pm 0.16$ vs $0.51 \pm 0.07, p<0.001$ ) in comparison to $S S c$ patients without DUs. PSV and RI of ulnar artery were significantly correlated with age $(\mathrm{p}=0.012$ and $p=0.019)$, disease duration ( $p=0.001$ and $p=0.001$ ) and Raynaud's phenomenon (RP) duration $(p=0.048$ and $p=0.028)$. PSV and $R I$ of tibial artery had significant correlation with age $(p=0.038$ and $p=0.009)$, systolic blood pressure $(p=0.022$ and $p=0.037)$ and diastolic blood pressure $(p=0.015$ and $p=0.010)$.

Conclusions: We concluded that digital ulceration in patients with SSc might be frequently related to the macrovascular dysfunction in below the elbow and knee. References:

[1] Walmsley D, Goodfield MJ. Evidence for an abnormal peripherally mediated vascular response to temperature in Raynaud's phenomenon. $\mathrm{Br} \mathrm{J}$ Rheumatol 1990;29:181-4.

[2] Chung L, Fiorentino D. Digital ulcers in patients with systemic sclerosis. Autoimmun Rev 2006;5:125-28.

[3] Ho M, Veale D, Eastmond C, Nuki G, Belch J. Macrovascular disease and systemic sclerosis. Ann Rheum Dis 2000;59:39-43.

[4] Veale DJ, Collidge TA, Belch JJ. Increased prevalence of symptomatic macrovascular disease in systemic sclerosis. Ann Rheum Dis 1995;54:853-5.

Disclosure of Interest: None declared

DOI: 10.1136/annrheumdis-2017-eular.1041

\section{AB0629 FROM LOCALIZED SCLERODERMA TO SYSTEMIC SCLEROSIS: A POSSIBLE EVOLUTION}

E. Cocchiara, C. Esposito, V. Raimondo, A. Spinella, F. Lumetti, M. Colaci, D. Giuggioli, C. Ferri on behalf of Rheumatology Unit, University of Modena and Reggio Emilia, Modena, Italy. Ospedale Policlinico di Modena, Modena, Italy

Background: Systemic Sclerosis (SSc) is a connective tissue disease characterized by skin fibrosis and visceral organ involvement. Localized Scleroderma (LoS), also known as morphea, is a fibrosing condition limited to the skin, subcutaneous tissue, underlying bone, and rarely central nervous system if present on face and head. SSc and LoS may share some aspects, such as histopathological findings, presence of autoantibodies and systemic symptoms, especially Raynaud phenomenon (RP). In this perspective they may represent two ends of a spectrum of disease.

Objectives: The aim of our study is to evaluate the evolution from LoS to SSc in our case series of SSc patients.

Methods: We retrospectively investigated 330 patients fulfilling the SScACR/EULAR criteria referred to our University-based Rheumatology Unit. The occurrence of LoS preceding the SSc diagnosis was evaluated for each patient on the basis of medical records; clinical, laboratory, and instrumental features were analyzed, from the presenting symptoms at the disease onset to the first visit and during the follow-up, with particular attention to very early cutaneous manifestations.

Results: Five SSc patients (1.5\% of our SSc series) had a clinical history of LoS prior to SSc diagnosis. All were women with mean age at time of LoS onset of $39 \pm 16.1 \mathrm{SD}$ years and time interval between LoS and SSc diagnosis of $19.2 \pm 16$ SD months. Skin biopsy was performed in $4 / 5$ patients showing nonspecific inflammatory infiltrate, collagen fiber deposition and dermis sclerosis. In all patients RP was the first extra-dermal symptom, preceding LoS in $2 / 5$ patients. Cutaneous involvement was represented by patches of skin sclerosis localized in limbs, trunk and face; while scleroderma was classified as cutaneous limited SSc in 4/5 patients and sine scleroderma in one. Following the SSc onset 2/5 patients had a history of digital ulcers, 1/5 esophagopathy, 1/5 interstitial lung disease; while capillaroscopy evidenced a SSc pattern in $4 / 5$ patients. ANA were detected in all patients with anti-ENA positivity in 3/5 (1 ACA, 1 anti-Scl70, 1 anti-U1RNP); the presence of autoantibodies was observed in $3 / 5$ individuals before SSc onset. None referred exposure to toxics or cigarettes smoke, while autoimmune thyropathy was the most frequent comorbidity. No local treatments had been employed for LoS but only low dosage of systemic steroids.

Conclusions: LoS and SSc are two distinct clinical entities that may share some clinical features; however, LoS is characterized by the absence of sclerodactyly, RP, digital ulcers, and typical SSc capillaroscopic changes; while possible internal organs involvement is much less frequently observed and the transition to SSC is exceptional and reported in only pediatric population. At our knowledge, this is the first observation of well-documented evolution from LoS to SSc in adult population as shown by updated review of the literature. The presence of RP and ANA positivity observed before the SSc onset can be considered as red flags of LoS evolution towards SSc, as reported in literature in pediatric population. SSc following LoS seems to be characterized by higher prevalence of vasculopatic symptoms compared to fibrotic complications. Finally, a careful clinical and laboratory monitoring of patients with LoS is recommendable to early identify the possible evolution to overt SSc.

Disclosure of Interest: None declared

DOI: 10.1136/annrheumdis-2017-eular.5120

\section{AB0630 CLINICAL SPECTRUM TIME COURSE COMPARISON BETWEEN PL-7, PL-12 AND EJ POSITIVE ANTISYNTHETASE SYNDROME}

E. Trallero Araguas ${ }^{1}$, A. Selva O'Callaghan ${ }^{1}$, C.A. Scire ${ }^{2}$, V. Codullo ${ }^{3}$, S. Castaneda ${ }^{4}$, N. Ortego Centeno ${ }^{5}$, J.A. Pereira da Silva ${ }^{6}$, C. Fiehn ${ }^{7}$, A. Schwarting ${ }^{8}$, A. Manfredi ${ }^{9}$, G. Emmi ${ }^{10}$, S. Barsotti ${ }^{11}$, O. Molberg $^{12}$,

A. Doria ${ }^{13}$, S. Parisi ${ }^{14}$, S. Scarpato ${ }^{15}$, N. Pipitone ${ }^{16}$, M. Piga ${ }^{17}$, I. Cavazzana ${ }^{18}$, F.J. Lopez-Longo ${ }^{19}$, J. Bachiller-Corral ${ }^{20}$, M. Benucci $^{21}$, J. Rojas-Serrano ${ }^{22}$, K. Triantafyllias ${ }^{23}$, N. Perez-Gomez ${ }^{24}$, G. Cagnotto ${ }^{25}$, F. Maurier ${ }^{26}$,

L. Quartuccio ${ }^{27}$, E. Bartoloni Bocci ${ }^{28}$, P. Tomietto ${ }^{29}$, R. Caporali ${ }^{3}$, F. lannone ${ }^{30}$, M.A. Gonzalez-Gay ${ }^{31}$, L. Cavagna ${ }^{3}$ on behalf of AENEAS collaborative group. ${ }^{1}$ Internal Medicine, Vall d'Hebron General Hospital, Barcelona, Spain;

${ }^{2}$ Reumatology, UOC Reumatologia, Azienda Ospedaliero Universitaria S. Anna, Ferrara; ${ }^{3}$ Rheumatology, University and Irccs Foundation Policlinico S. Matteo, Pavia, Italy; ${ }^{4}$ Rheumatology, Hospital Universitario de la Princesa, IIS-IP, Madrid; ${ }^{5}$ Systemic Autoimmune Diseases Unit, Hospital Clínico San Cecilio, Granada, Spain; ${ }^{6}$ Rheumatology, Centro Hospitalar e Universitário, Coimbra, Portugal; ${ }^{7}$ Rheumatology, ACURA Centre, Baden Baden; ${ }^{8}$ Rheumatology, University Hospital Johannes-Gutenberg, Mainz, Germany; ${ }^{9}$ Rheumatology, Azienda Ospedaliero-Universitaria Policlinico, Modena; ${ }^{10}$ Experimental and Clinical Medicine, University, Florence; ${ }^{11}$ Rheumatology, AOU Pisana, Pisa, Italy;

${ }^{12}$ Rheumatology, University Hospital, Oslo, Norway; ${ }^{13}$ Rheumatology, University, Padua: ${ }^{14}$ Rheumatology, Città della salute e della scienza, Torino;

${ }^{15}$ Rheumatology, ASL Salerno, Scafati; ${ }^{16}$ Rheumatology, S.Maria Hospital -IRCCS, Reggio Emilia; ${ }^{17}$ Rheumatology, University, Cagliari; ${ }^{18}$ Rheumatology, University and AO Spedali Civili, Brescia; ${ }^{19}$ Rheumatology, Hospital General Universitario Gregorio Marañón, Madrid, Italy; ${ }^{20}$ Rheumatology, University Hospital Ramón y Cajal, Madrid, Spain; ${ }^{21}$ Rheumatology, Azienda Sanitaria di Firenze, S. Giovanni di Dio Hospital, Firenze, Italy; ${ }^{22}$ Rheumatology, Instituto Nacional de Enfermedades Respiratorias, Ismael Cosío Villegas, Mexico city, Mexico; ${ }^{23}$ Rheumatology, ACURA Centre, Bad Kreuznach, Germany;

${ }^{24}$ Rheumatology, Hospital Clinico Universitario, Santiago de Compostela, Spain;

${ }^{25}$ Rheumatology, Skane University Hospital, Lund, Sweden; ${ }^{26}$ Medicine, HPMetz, Metz, France; ${ }^{27}$ Rheumatology, Santa Maria della Misericordia Hospital, Udine;

${ }^{28}$ Rheumatology, University, Perugia; ${ }^{29}$ Rheumatology, Cattnara Hospital, Trieste; ${ }^{30}$ Rheumatology, University, Bari, Italy; ${ }^{31}$ Rheumatology, Hospital Universitario Marqués de Valdecilla, IDIVAL, University of Cantabria, Santander, Spain

Background: Arthritis, myositis and Interstital lung disease (ILD) represent the classic clinical triad of antisynthetase syndrome (ASSD). In anti Jo-1 positive patients, these findings may appear also during the follow-up. Even if a similar cumulative trend has been showed also in non anti Jo-1 positive ASSD, a head to head comparison of clinical spectrum time course in these patients is still lacking Objectives: To assess the clinical spectrum time course in non anti Jo-1 positive ASSD, according to different underlying non anti Jo-1 specificities

Methods: Clinical, laboratory and instrumental data collection of anti PL-7, PL-12, and EJ positive patients from an international database of ASSD

Results: We identified 63 (42\%) anti PL-7, 66 (44\%) anti PL-12 and 20 (14\%) anti EJ positive patients, reporting their characteristics in table 1 (disease onset) and 2 (last follow-up). At disease onset, no substantial differences were observed. At the end of follow-up, we observed some differences between anti PL-12 and both anti PL-7 and anti-EJ positive patients. In particular, anti PL-12 positive patients presented less frequently ex-novo triad findings and had a reduced prevalence of 
myositis. From the clinical point of view, the main pattern of disease presentation was an isolated ILD in all groups at the onset and only in anti-PL12 positive ASSD at last follow-up.
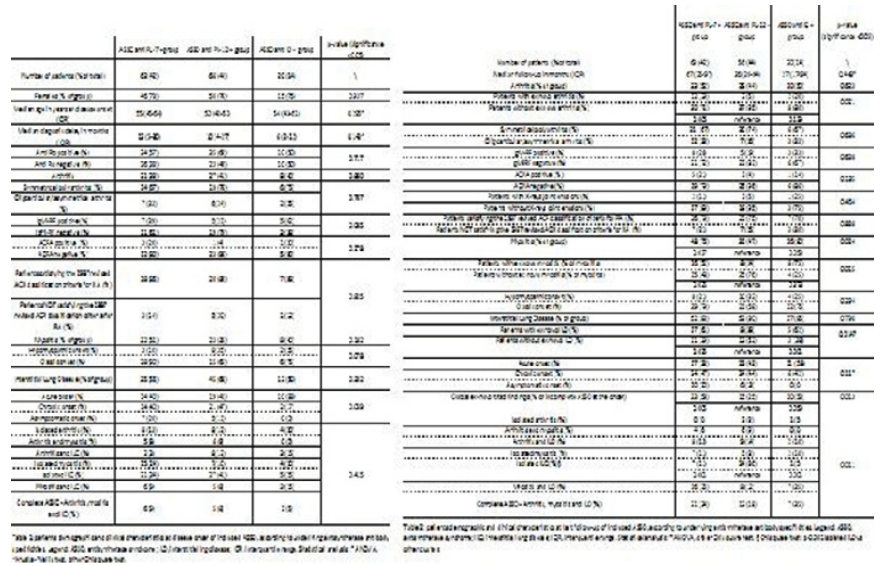

Conclusions: Our study seems to indicate that clinical spectrum time course of anti PL-12 positive ASSD is different from that of anti PL7 and of anti EJ positive ASSD. The clinical pattern associated with these two latter antibodies was very similar. Furthermore, anti PL-12 positive patients seems to have a more stable disease, with a less common occurrence of ex-novo triad findings during the follow-up

References:

[1] Cavagna L. Medicine 2015.

[2] Cavagna L. CRAI 2016.

[3] Cavagna L. ARD 2016 (Abstract).

[4] Trallero Araguas E. Scand J Rheumatol 2016

Acknowledgements: To all members of the AENEAS collaborative group.

Disclosure of Interest: None declared

DOI: 10.1136/annrheumdis-2017-eular.2592

\section{AB0631 THE CLINICAL CONSEQUENCES PRESENCE OF ANTI-PM/SCL ANTIBODIES IN SYSTEMIC SCLEROSIS}

E. Wielosz, M. Dryglewska, M. Majdan. Department of Rheumatology and Connective Tissue Diseases, Medical University of Lublin, Lublin, Poland

Background: Anti-PM/Scl (a-PM/Scl) antibodies are found in different systemic autoimmune disease such as polymyositis, dermatomyositis, systemic sclerosis (SSc), and overlap syndromes. According to literature they are detected in about $2 \%$ of patients with SSc, but their presence are more common in SSc with myositis overlap. Features positively associated with the presence of a-PM/Scl antibodies included younger age at disease onset, skeletal muscle involvement, calcinosis, inflammatory arthritis, and overlap disease. On the other hand interstitial lung disease and gastrointestinal symptoms were less frequent in SSc patients with a-PM/Scl.

Objectives: The aim of the study was to assess the clinical consequences presence of a-PM/Scl antibodies in patients with SSc.

Methods: The study was performed in 126 European Caucasian SSc patients (98-female and 28-male) hospitalized consecutively in the Department of Rheumatology and Connective Tissue Diseases. Patients fulfilled the ACR classification criteria of SSc (59 have diffuse cutaneous SSc and 67 limited SSc). The study group were studied according to the presence of a-PM/Scl antibodies applying commercial test - EUROLINE Systemic Sclerosis Profile. Detection and interpretation of results was carried out electronically using the specific program Euroimmun- EUROLINEScan. The subtype of SSc, incidence of internal organ involvement and serological profile were determined in the whole group. Due to the presence of a-PM/Scl antibodies, patients were divided into two groups a-PM/Scl (+) SSc - 22 pts and a-PM/Scl (-) SSc- 104 pts.

Results: a-PM/Scl antibodies were detected in $22 / 126$ patients with SSc $(17,5 . \%)$ We showed a significant positive association with a-PM/Scl antibodies and myalgia or myosistis $(p=0,0379)$, contractures $(p=0,0002)$ and prevalence of overlap syndrome $(p=0,0142)$. There were no relationship between the presence of a-PM/Scl antibodies and subtypes of SSc, other organ involvement, digital ulcers or calcinosis.

Conclusions: In SSc anti-PM/Scl antibodies are frequently associated with myalgia or myositis, contractures and overlap syndrome.

Disclosure of Interest: None declared

DOI: 10.1136/annrheumdis-2017-eular.4393

\section{AB0632 ASSESSMENT OF VEGF AND OTHER CYTOKINES IN THE TEAR OF PATIENTS WITH SYSTEMIC SCLEROSIS}

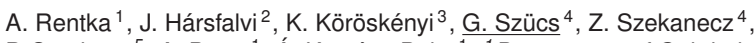
P. Szodoray ${ }^{5}$, A. Berta ${ }^{1}$, Á. Kemény-Beke ${ }^{1}$. ${ }^{1}$ Department of Ophthalmology, University of Debrecen, Faculty of Medicine, Debrecen; ${ }^{2}$ Department of Biopsysics and Radiation Biology, Semmelweis University, Budapest; ${ }^{3}$ Department of Biochemistry and Molecular Biology; ${ }^{4}$ Department of Rheumatology, University of Debrecen, Faculty of Medicine, Debrecen, Hungary; 5 Institute of Immunology, Rikshospitalet, Oslo, Norway

Background: Systemic sclerosis (SSc) is an autoimmune disease, characterized by widespread small vessel vasculopathy, immune dysregulation with production of autoantibodies, and progressive fibrosis. SSc may be associated with sicca syndrome. Changes in levels of proangiogenic and proinflammatory cytokines had already been determined largely in serum, however, the local inflammatory and cytokine milieu in the tear of SSc patients has not yet been evaluated.

Objectives: We wished to determine VEGF and other cytokine and chemokine levels in tear samples of SSc patients.

Methods: First, forty-three patients (40 female and 3 men, mean (SD) age 61 (48-74) years) with SSc and 27 healthy controls were enrolled in the VEGF study. Basal tear sample collection and tear velocity investigations were carried out followed by an ophthalmological examination. Total protein concentrations and VEGF levels were determined in tear samples. In the multiple cytokine study, unstimulated tear samples were collected from nine patients with SSc and 12 age- and gender-matched healthy controls. The relative levels of 102 different cytokines were determined by a cytokine array, and then absolute levels of four key cytokines were determined by a magnetic bead assay.

Results: In the first study, the mean collected tear fluid volume developed 10.4 $L(1.6-31.2)$ in patients and $15.63 \mathrm{~L}(3.68-34.5)$ in control subjects. The mean total protein level was $6.9 \mathrm{~g} / \mathrm{L}(1.8-12.3)$ and $4.1 \mathrm{~g} / \mathrm{L}(0.1-14.1)$ in tear samples of SSc patients and controls, respectively. In patients with SSc, the mean VEGF tear concentration was $4.9 \mathrm{pg} / \mathrm{L}(3.5-8.1)$ compared to $6.15 \mathrm{pg} / \mathrm{L}(3.84-12.3)$ in healthy samples. Multicytokine-array studies revealed shifted cytokine profile characterized by predominance of proinflammatory mediators in the tear samples of SSc patients. Out of the 102 analyzed proteins, nine were significantly increased in tears of patients with SSc. Based on the multiplex bead results, CRP, interferon-inducible protein 10 (IP-10) and monocyte chemoattractant protein-1 $(\mathrm{mcp}-1)$ levels were significantly higher in tears of patients with SSc compared to controls.

Conclusions: Impaired angiogenesis has been found by other investigators in SSc. This is reflected by lower VEGF levels in the tear samples of SSc patients compared to controls. The multi-cytokine array study revealed increased production of CRP and two important pro-inflammatory chemokines in the tear of SSc patients. Our current data depict a group of inflammatory mediators, which may play a significant role in ocular pathology of SSc.

Disclosure of Interest: None declared

DOI: 10.1136/annrheumdis-2017-eular.3218

\section{AB0633 RITUXIMAB IN SCLERODERMA RELATED INTERSTITIAL LUNG DISEASE: A SINGLE CENTRE EXPERIENCE FROM NORTH INDIA}

G. Naidu, S.K. Sharma, V. Dhir, S. Jain. Internal Medicine, PGIMER,

\section{Chandigarh, India}

Background: Pulmonary involvement is one of the major causes of morbidity and mortality in patients with progressive systemic sclerosis (PSS). Clinically significant interstitial lung disease (ILD) is noted in $25 \%$ of patients and accounts for $33 \%$ of deaths in PSS patients $[1,2]$. Cyclophosphamide (CYC) and mycophenolate mofetil (MMF) have been shown to retard progression of ILD [3, 4]. The limited data on rituximab indicate that rituximab might be effective in PSS related ILD [5].

Objectives: To study the efficacy of rituximab in patients with PSS related ILD.

Methods: The clinical details of all patients of PSS related ILD who were treated with rituximab were noted retrospectively from the case files. Forced vital capacity (FVC) value before and 1 year after administration of rituximab were noted. Increase in FVC by $10 \%$ from baseline was considered as improvement and fall in FVC by $10 \%$ or absolute value less than $40 \%$ of predicted was considered as worsening. Patients with FVC $\pm 10 \%$ from baseline were considered to have stabilized lung functions.

Results: A total of 11 patients received rituximab between 2013 and 2016 Six $(54.5 \%)$ patients were females. Median age of the subjects was 44 years (range: $31-75$ years). All patients received intravenous CYC at least 1 year before rituximab administration and had either not responded to CYC or worsened after initial response to CYC. All patients received 2 doses of rituximab (1gram each) at 2 weeks interval. Median FVC before rituximab was $57 \%$ of predicted. Two patients did not have post rituximab FVC values. Median FVC 1 year after rituximab was $54 \%$ predicted. Out of the remaining 9 patients, $2(22.2 \%)$ patients had improvement in FVC, 6 (66.7\%) patients had stabilization of FVC and 1 patient worsened. One patient, who had stabilization of FVC with rituximab expired after 2 years of receiving rituximab.

Conclusions: Rituximab was effective in stabilization of lung functions in patients of PSS with ILD who did not have favourable outcome with intravenous CYC. 